\title{
A Model for Sharing of Confidential Provenance Information in a Query Based System
}

\author{
Meiyappan Nagappan and Mladen A.Vouk \\ North Carolina State University, \\ Raleigh, NC 27695, USA \\ \{mnagapp, vouk\}@ncsu.edu
}

\begin{abstract}
Workflow management systems are increasingly being used to automate scientific discovery. Provenance meta-data is collected about scientific workflows, processes, simulations and data to add value. There is a variety of workflow management tools that cater to this. The provenance information may have as much value as the raw data. Typically, sensitive information produced by a computational processes or experiments is well guarded. However, this may not necessarily be true when it comes to provenance information. The issue is how to share confidential provenance information. We present a model for sharing provenance information when the confidentiality level is decided by the user dynamically. The key feature of this model is the Query Sharing concept. We illustrate the model for workflows implemented using provenance enabled Kepler system.
\end{abstract}

Keywords: Provenance, Confidentiality, Workflow management tools.

\section{Introduction}

The provenance collection approach in workflow support systems can be flow based, annotation based, or a combination of both [4. Workflow systems execute scientific simulations and secure the output data in order to maintain confidentiality/privacy and ownership of the sensitive data. Most of them, however, do not have any mechanisms in place for maintaining the confidentiality of provenance information. Here we use the term confidentiality as defined in ISO/IEC17799[11]: ensuring that information is accessible only to those authorized to have access.Building the provenance collection system with such a mechanism should be the priority from the very beginning [13. Security and confidentiality must be considered in an integrated context. For example, one must implement security to ensure confidentiality of the information. Security is a process or tactics that ensures that the desired level of confidentiality can be an outcome [9].

While confidentiality of provenance information is important, so is the sharing of information among collaborators. Most of the scientific projects are highly collaborative projects. Often the collaborators are in different research labs in the country, and sometimes even in different countries. They may be working 
together on the same approach, or may be taking different approaches to scientific discovery. Provenance data throws a lot of light onto a particular scientific problem, process, and the data it produces. It can discover a knowledge nugget that needs to be shared among all collaborators, and some that need to be shared sparingly. Hence provenance information of these projects is unlike any other data-centric application. Provision of mechanisms for confidentiality, like the ones in other similar system, should not restrict the sharing of provenance data with trusted collaborators. For example, scientist A is the owner of runs 1,2 , and 3 of a scientific simulation. Each of the runs produced a set of provenance data viz. R1, R2 and R3 respectively. User A wants to share subsets of R1, R2 and R3, with Scientists B and C. Each of these subsets can be different from the others. An appropriate mechanism should enable easy sharing of data either on a per run basis, or on a per user group basis, or individually. The goal of the current work is to develop a model, in the context of the provenance for scientific simulations, that

- Enables an easy sharing of the provenance data.

- Does not compromise the confidentiality of the provenance data.

- Allows for dynamic changes in the confidentiality levels.

The specific focus is on the systems that use Kepler [1 for scientific workflow automation and management. Kepler workflows are composed of a set of actors (processes) forming, in more complex situations, generalized activity networks [3], 8]. The order of execution of these actors depends on the nature of the problem (workflow) being solved and the Model of Computation (MoC) used to execute the workflow [6], [12]. These MoC's (or process schedulers) are called directors in Kepler. One version of Kepler implements a provenance collection mechanism [3], 12. We describe it in more detail in Section 2.

\section{Provenance in Kepler}

The workflow support system we have been using is Kepler 1]. There is a version of Kepler that directly supports provenance recording. The Kepler Provenance Recorder (PR) is described in [3], 6], 12. PR implements the Read-Write-State reset (RWS) trace information (flow-based provenance) first introduced in workflow systems in [6]. The recorder captures the flow of data objects between the ports of actors. The reads(consume), and writes(emit) done by an actor are captured as provenance. Also, events such as the 'flushing' of the state of the actors is captured. It generates a unique token id for every token consumed/emitted by an actor. Each execution of the workflow is assigned a unique id too. An implementation model is described in section 4. The PR is designed so that we need not edit any of the Kepler actors. It is similar to a Kepler director in that it is configured in the same way, but it is different in the fact that it does not control the workflow, rather it just listens to it (in that sense it is more akin to the Kepler debugging facilities that allow detailed workflow tracing). When the Kepler PR actor is included into a workflow, it automatically collects the provenance data by listening to the ports of all the actors in the workflow. 
We propose to slightly modify the RWS relational tables in order to achieve our goal. The relational tables in our model are: users Table (username, workflow name, run id, annotation), actorTable (run id, actor id, port id, annotation), traceTable (port id, token, event, annotation), tokenTable (token, object, annotation), objectTable( object, value, type, annotation). This is very similar to the provenance relations described in [12. The difference is that here we have introduced an annotation field in each relation and the users Table relation for keeping tack of the owner of a run. This enables capture of the provenance that was recorded by their RWS PR. However, the annotation field in each of the above relations will get its value from the user only, and not from Kepler.

\section{An Implementation Model}

In this section we describe a model that can accommodate the sharing of confidential provenance information in a scenario where the confidentiality level changes dynamically. Here we try to reach the goal stated above through five sub goals. The Model is being implemented in a system being built by DOE Scientific Data Management Center [2], 17]. Fig. 1] illustrates the architecture. The details of this figure are discussed in the following text.

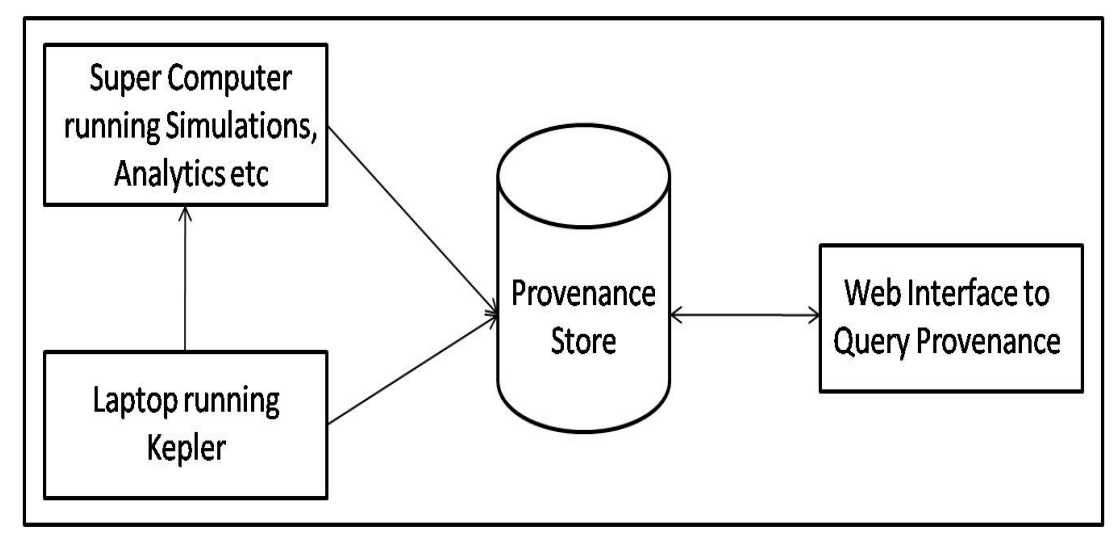

Fig. 1. Top-level architecture view of our Model

\subsection{Sub Goal 1: Data Ownership}

We need to ensure that the person who generates the simulation data would be the owner of the original provenance data. To achieve this we use a three tiered (Client-Application Logic-Database) approach and build role based access control in the application logic layer. When collecting provenance the client would either be the workflow management system or the scripts and simulation applications running in the super computer, or other affiliated analytics resources. The recording API is the application logic layer and makes sure that the information from the clients are stored in the appropriate tables of the schema. Also 
there is an authentication service that verifies the user. Thus the data in the provenance schema is indexed by the user and a particular run id.

In order to view this data, we use a Web Application (WA). Here the client is the Web Interface (WI) and the Query API is the application logic. Here too we have an authentication service that will authorize the user. Since the data is indexed by user, the query API is able to fetch a particular user's data. Each user has multiple runs under their username [5]. They pick the one they want to see the provenance data for. The query API will execute some of the default queries only on the dataset for this particular run and for this particular user. Then the user who launched this query can refine the dataset as they please. This is similar to the role based access available in many database applications.

\subsection{Sub Goal 2: Editing and Audit Trail}

The access privileges to the database are restricted to prevent any unwarranted use of provenance data. Once the data is created, users cannot delete or update any of the provenance information. But they can modify annotation fields of the records in the provenance relations and the queryTable relation. The prevention of edits to the data is important not just for audit purposes. Another major reason is to maintain consistency. A collaborator should not get a different dataset when he/she executes shared queries at different times. This can be possible only if the owner is not allowed to delete any provenance data. Only an administrator may delete this data. But all superuser actions also must be logged. A trail for this must be maintained for audit purposes. A system that tracks the changes that happen to database relations would suffice for this purpose. Even though users cannot delete the provenance data they can, through the annotation fields, comment on the accuracy of the provenance data.Thus by using access privileges in modern database systems we are able to achieve this sub goal.

\subsection{Sub Goal 3: Data Annotation}

The annotation field in the queryTable relation and the other relations may be updated from the WA. They can annotate the datasets regarding any inaccuracies or interesting findings. They may update only the annotation field in the provenance relations and the queryTable relation. The users when sharing can choose not to share the annotations. Annotation in itself is a form of user specified meta-data. Thus annotations help in differentiating the useful provenance information without deleting the inaccurate data, as well as add user level meta data to the results. Thus through the WA we are able to achieve this sub goal.

\subsection{Sub Goal 4: Data Sharing}

The ability of researchers to share their provenance data with their colleagues in an easy manner is probably as important as the confidentiality of this data. We introduce the notion of query sharing for this purpose. A logged-in user will be able to see data in the WI of the WA. If the scientist finds the provenance data 
that they currently see as interesting and wants to share it, he/she can do so by saving the query that created that dataset and sharing it with collaborators. This is analogous with the WYSIWYG concept. Here it is: What You See Is What You Want to Share(WYSIWYWTS): The relation queryTable (Query ID, Saved by, Saved for, Query, Timestamp, Allow Cascading, Revoke Active) is used to save the queries, and the relation annotTable (UserID, Query ID, Annotation, Viewable) is used to store the annotation for the data set that is to be shared. The WA provides saving of the query that created the dataset. The scientist who wants to share the data can choose to annotate the dataset. For example if User U1 wants to save the dataset of run Rx and share it with User U2, then the entry in the queryTable relation would look like this: (QID, U1, U2, Select Query similar to the above one, timestamp, A binary 1/0 for whether cascading of should be allowed or not, A binary 0/1 if the collaborator has access currently or not). They can also choose to annotate the dataset by making an entry in the annotTable relation. A typical entry would be (UID, QID, Any relevant annotation to the dataset, $A$ binary $1 / 0$ if the collaborator should see the annotation or not)

The collaborator, when logged in to the WA, will be able to see, give the right access rights, all their data in the hierarchical fashion above. In a separate tab they will also be able to see the queries (possibly not the actual query itself, but rather the Query ID, annotation, timestamp, saved by and saved for columns). This is done by the WA which will pass the user-id of the currently logged-in user and one of the Query API's will fetch all the entries in the queryTable where the user id matches the user id in the saved by or saved for columns and the corresponding entries in the annotTable if the viewable attribute is set to 1 .

A user selects a query to run. Then the user sees the data. If the user wants to refine the query the user can do so as required. The queries are built for refining acts only on the dataset that was shared and not on the whole database. By abstracting the execution of the query to an API that will manage whose data is to be accessed, we restrict the user from seeing data that is not meant to be seen. As an extra measure, we could encrypt the query attribute of the queryTable relation in the database, to protect the data in case the queryTable in the database gets compromised. The saved query is not shown to the users and can be use in an execute-only mode.

The cascade attribute in the queryTable is used either allow or deny a collaborator from passing the shared information to another person. The revoke attribute is used to remove access to a particular query from a particular user by the owner of that query. Also they can save interesting subsets of the provenance information for themselves too. In this case saved by and saved for attributes in the queryTable will be the same as the owner. A collaborator in our model though may not be able to annotate individual pieces in a shared dataset. They can only annotate the dataset as a whole. For this they would make an entry into the annotTable. If they want the owner to view their annotations then they would set the viewable attribute to 1 . 
A user can thus give others access to all their data, or to a particular dataset, or a particular part of a particular dataset. Thus the granularity to which the user wants to share data depends on the user. Each time the user can decide to share a different piece of the dataset without editing any rule set for access control. Also the entire chosen dataset can be shared by saving a single query instead of sharing each record in the dataset or saving a copy of the whole dataset. Thus with the help of the Query Sharing concept we are able to achieve the fourth sub goal too.

As we can see, the query sharing concept is very similar to stored procedures. Both have the same overhead. But by saving queries in a table we extend their scope to add more meta-data to the saved query which would not be possible in stored procedures. Now, let us discuss why the data sharing concept would be better than any other solution given the following constraints:

- Dynamically decide what to share: Due to this constraint we are not able to build the logic of the query in the application layer. Since applications are static, the users would not be able to able to add a new query for a subset of the dataset that should be shared.

- Size of the set of information to be shared is large: Since the datasets are large, and so are the subsets of information, we may not be able to share copies with the collaborators.

- Subset of information rather than individual records: The data users share are subsets. If we were to use a high level language to control access, then the users would have to individually pick the cells in the subset that they want to share. The large size, and the fact that they can be thought of as atomic piece of information, would make the process of individually picking cells too time consuming.

Thus given these constraints our query sharing solution scales well(as only one row is added for each subset to be shared), and also it is very flexible(just a click away from sharing a subset).

\subsection{Sub Goal 5: Data Audit and Verification}

The inclusion of auditing in the model is to maintain accuracy of the data. Users with malicious intent may not tamper with the data if they know that it is going to be audited and that auditors have sufficient information to find any such use. Thus authenticated auditors are users who can view the original data, provenance data, and annotations. They are to be provided with sufficient information for auditing. The provenance relations have annotations in them for the auditors to verify the data against. Also the queryTable relation has timestamps in it for the auditors to find any discrepancy in the sharing of data. Finally since all the edits to the database had a trail, any missing information can be accounted for by the auditors. Thus by collecting and having access to all this information the auditors can catch any malicious use of data.

Hence by meeting these five sub goals we are ale to achieve the main goal of sharing provenance information at any level of confidentiality. 


\section{Limitations and Conclusions}

The implementation model we have presented has some limitations. For example, it is query-centric. Only provenance systems that store data in databases and fetch them using queries can use this approach effectively. Another limitation is that the model requires automatic run-time provenance collection. The user is still allowed to annotate the data and create manual provenance, but not to modify original records. This provides a considerable level of integrity for the originally (and automatically) collected data, but perhaps less oversight for manually added annotations. Also to be noted is that only owners of the provenance data can freely annotate any part of the dataset. The collaborators can only annotate the entire shared subset and not any individual part in it. Even in automatic provenance collection systems we could face the security and privacy problems discussed in [7].

With more and more emphasis being laid on provenance data collection in scientific workflow applications [14, [15], the issue of sharing provenance with varying levels of confidentiality becomes increasingly important. We believe that the simple model described in this paper is able to ensure considerable level of confidentiality of provenance data, as well as sharing of it amongst trusted collaborators. The data sharing technique described in this paper allows for users to change the level of collaboration dynamically. This model addresses the integrity, confidentiality and availability, and ownership responsibility issues raised in [10, and 18. But this model does not address the issues arising due to long term storage and scalability of provenance data discussed in [18. There are some systems like PASS [16] that are used to provide security using provenance data. But there is not much research in the field of securing provenance data, providing confidentiality and enforcing privacy policies.

\section{Acknowledgments}

This project is funded in part by the DOE SciDAC grant DE-FC02-01ER25809 and the IBM SUR program. I would like to thank the SPA group of the SDM Center for their collaboration. I would also like to thank Dr.Yu and Dr.Sherriff, and graduate students Vinod Arjun, Lucas Layman, Aaron Massey, and Andy Meneely for discussions and insights into the various aspects of this research.

\section{References}

1. Kepler development and download site, http://kepler-project.org/

2. Scientific Data Management Center,http://sdm.lbl.gov/sdmcenter/index.html

3. Altintas, I., Barney, O., Jaeger-Frank, E.: Provenance Collection Support in the Kepler Scientific Workflow System. In: Moreau, L., Foster, I. (eds.) IPAW 2006. LNCS, vol. 4145, pp. 118-132. Springer, Heidelberg (2006)

4. Barga, R.S., Digiampietri, L.A.: Automatic Generation of Workflow Provenance. In: Moreau, L., Foster, I. (eds.) IPAW 2006. LNCS, vol. 4145, pp. 1-9. Springer, Heidelberg (2006) 
5. Barreto, R., Critchlow, T., Khan, A., Klasky, S., Kora, L., Ligon, J., Mouallem, P., Nagappan, M., Podhorszki, N., Vouk, M.: Managing and Monitoring Scientific Workflows through Dashboards. In: Poster \# 93, at Microsoft eScience Workshop Friday Center, University of North Carolina, Chapell Hill, NC, October 13 - 15, p. 108 (2007)

6. Bowers, S., McPhillips, T., Ludeascher, B., Cohen, S., Davidson, S.B.: A Model for User-Oriented Data Provenance in Pipelined Scientific Workflows. In: Moreau, L., Foster, I. (eds.) IPAW 2006. LNCS, vol. 4145, pp. 133-147. Springer, Heidelberg (2006)

7. Braun, U., Garfinkel, S., Holland, D.A., Muniswamy-Reddy, K.-K., Seltzer, M.I.: Issues in Automatic Provenance Collection. In: Moreau, L., Foster, I. (eds.) IPAW 2006. LNCS, vol. 4145, pp. 171-183. Springer, Heidelberg (2006)

8. Elmaghraby, S.E.: Activity Networks: Project Planning and Control by Network Models. Wiley-Interscience, New York (1977)

9. Griffiths, P.P., Wade, B.W.: An authorization mechanism for a relational database system. ACM Transactions on Database Systems 1(3), 242-255 (1976)

10. Hasan, R., Sion, R., Winslett, M.: Introducing secure provenance: problems and challenges. In: Proceedings of the 2007 ACM workshop on Storage security and survivability, pp. 13-18. ACM, Alexandria (2007)

11. ISO/IEC 17799. Information technology - Security techniques - Code of practice for information security management (2000) (Rev. 2005), http://www.iso.org/ iso/en/prods-services/popstds/informationsecurity.html

12. Ludaescher, B., Podhorszki, N., Altintas, I., Bowers, S., McPhillips, T.: From Computation Models to Models of Provenance: The RWS Approach. Concurrency and Computation: Practise and Experience 20(5), 507-518

13. McGraw, G.: Building secure software: better than protecting bad software. Software, IEEE 19(6), 57-58

14. Moreau, L., Foster, I.: IPAW 2006. LNCS, vol. 4145. Springer, Heidelberg (2006)

15. Moreau, L., Ludäscher, B.: Concurrency and Computation: Practice \& Experience - Special Issue on the First Provenance Challenge. Wiley, Chichester (2007)

16. Muniswamy-Reddy, K.-K., Holland, D.A., Braun, U., Seltzer, M.I.: Provenance Aware Storage Systems. In: Proceedings of the 2006 USENIX Annual Technical Conference, p. 4 (June 2006)

17. Nagappan, M., Altintas, I., Chin, G., Crawl, D., Critchlow, T., Koop, D., Ligon, J., Ludaescher, B., Mouallem, P., Podhorszki, N., Silva, C., Vouk, M.: Provenance in Kepler-based Scientific Workflow Systems. In: Poster \# 41, at Microsoft eScience Workshop Friday Center, University of North Carolina, Chapell Hill, NC, October 13 - 15, p. 82 (2007)

18. Tan, V., Groth, P., Miles, S., Jiang, S., Munroe, S., Tsasakou, S., Moreau, L.: Security Issues in a SOA-Based Provenance System. In: Moreau, L., Foster, I. (eds.) IPAW 2006. LNCS, vol. 4145, pp. 203-211. Springer, Heidelberg (2006) 